TRANSACTIONS OF THE

AMERICAN MATHEMATICAL SOCIETY

Volume 365, Number 5, May 2013, Pages 2469-2488

S 0002-9947(2012)05708-4

Article electronically published on September 18, 2012

\title{
IMMERSED SURFACES AND SEIFERT FIBERED SURGERY ON MONTESINOS KNOTS
}

\author{
YING-QING WU
}

\begin{abstract}
We will use immersed surfaces to study Seifert fibered surgery on Montesinos knots, and show that if $\frac{1}{q_{1}-1}+\frac{1}{q_{2}-1}+\frac{1}{q_{3}-1} \leq 1$, then a Montesinos knot $K\left(\frac{p_{1}}{q_{1}}, \frac{p_{2}}{q_{2}}, \frac{p_{3}}{q_{3}}\right)$ admits no atoroidal Seifert fibered surgery.
\end{abstract}

\section{INTRODUCTION}

Exceptional Dehn surgeries on arborescent knots have been studied extensively. They have been classified for arborescent knots of length at least $4 \mathrm{Wu2}$, as well as for all 2-bridge knots [BW]. There is no reducible surgery on hyperbolic arborescent knots Wu1, and toroidal surgeries on length 3 Montesinos knots have also been classified [Wu3]. Therefore atoroidal Seifert fibered surgeries on Montesinos knots of length 3 are the only ones on arborescent knots that have not been determined.

Atoroidal Seifert fibered surgery is much more difficult to deal with than other types of exceptional surgeries. For example, the minimum upper bounds for distances between two types of exceptional Dehn fillings on hyperbolic manifolds have all been determined when those are not atoroidal Seifert fibered, but no such bound is known when one of them is. See GW] and the references therein for works in that direction. The major difficulty in dealing with atoroidal Seifert fibered surgery is that there is no embedded essential small surfaces (sphere, disk, annulus, or torus) in such manifolds, and therefore one cannot use those traditional combinatorial methods on intersection graphs for such surgery problems. Those with infinite fundamental group do contain immersed essential tori, but there had not been much success using them as tools in solving Dehn surgery problems. In this paper, however, we will use those immersed surfaces as a major tool. We will study the intersection of an immersed surface $F$ with the tangle decomposition surfaces and the tangle spaces, and show that no such surface could exist when the tangles are not too simple.

A length 3 Montesinos knot $K\left(\frac{p_{1}}{q_{1}}, \frac{p_{2}}{q_{2}}, \frac{p_{3}}{q_{3}}\right)$ is the cyclic union of three rational tangles $T\left(\frac{p_{i}}{q_{i}}\right)$, where $q_{i} \geq 2$ and $p_{i}, q_{i}$ are coprime. The integer part of the $p_{i} / q_{i}$ can be shifted around, so we will always assume that $2\left|p_{i}\right| \leq q_{i}$ for $i=1,2$. Given a knot $K$ in $S^{3}$, we use $K(r)$ to denote the manifold obtained by Dehn surgery on

Received by the editors April 23, 2011 and, in revised form, September 3, 2011.

2010 Mathematics Subject Classification. Primary 57N10.

Key words and phrases. Immersed surfaces, Dehn surgery, Seifert fibered manifolds, Montesinos knots.

(C) 2012 American Mathematical Society Reverts to public domain 28 years from publication 
$K$ along a slope $r$ on $\partial N(K)$, where $N(K)$ is a tubular neighborhood of $K$. The following is our main theorem.

Theorem 1.1. Suppose $K=K\left(\frac{p_{1}}{q_{1}}, \frac{p_{2}}{q_{2}}, \frac{p_{3}}{q_{3}}\right)$ is a hyperbolic Montesinos knot of length 3. If $\frac{1}{q_{1}-1}+\frac{1}{q_{2}-1}+\frac{1}{q_{3}-1} \leq 1$, then $K$ admits no atoroidal Seifert fibered surgery.

Recently Ichihara and Jong [IJ2] showed that the only toroidal Seifert fibered surgery on Montesinos knots is the 0 surgery on the trefoil knot, hence the theorem is still true with the word "atoroidal" deleted. By Wu1 there is no reducible surgery on hyperbolic Montesinos knots, so the following result follows immediately from Theorem 1.1 and the classification of toroidal surgeries on these knots $\mathrm{Wu} 3$, Theorem 1.1].

Corollary 1.2. Suppose $K=K\left(\frac{p_{1}}{q_{1}}, \frac{p_{2}}{q_{2}}, \frac{p_{3}}{q_{3}}\right)$ is a hyperbolic Montesinos knot of length 3. If $\frac{1}{q_{1}-1}+\frac{1}{q_{2}-1}+\frac{1}{q_{3}-1} \leq 1$ and a Dehn surgery $K(r)$ is nonhyperbolic, then $\left|p_{i}\right|=1, r$ is the pretzel slope, and $K(r)$ is toroidal.

We may assume $2 \leq q_{1} \leq q_{2} \leq q_{3}$. Corollary 1.2 and $\mathrm{BW}$, Wu2 provide a classification of exceptional Dehn surgeries on all arborescent knots except those $K\left(\frac{p_{1}}{q_{1}}, \frac{p_{2}}{q_{2}}, \frac{p_{3}}{q_{3}}\right)$ with $q_{1}=2$, or $\left(q_{1}, q_{2}\right)=(3,3)$, or $\left(q_{1}, q_{2}, q_{3}\right)=(3,4,5)$. Further restrictions on $p_{i}$ and the surgery slopes for these cases will be given in Theorem 8.2.

Theorem 1.1 is known in the special case that $K(r)$ has finite fundamental group. The classification of finite surgeries on Montesinos knots has been completed by Ichihara and Jong [IJ1. It used the results of Delman De on essential laminations, Mattman's result $\mathrm{Ma}$ on surgery on pretzel knots, and Ni's result $\mathrm{Ni}$ on Heegaard Floer homology and fibered knots. See also [Wa] and [FIKMS]. While our major goal is to prove Theorem 1.1 for infinite Seifert fibered surgeries, we will also provide an independent proof of it for the finite surgery case. We extend the thin position idea of Gabai $\mathrm{Ga}$ and use an immersed thin sphere to replace the immersed essential torus in the case that $K(r)$ has infinite fundamental group. This works fine in our setting and sometimes the proof is simpler than for infinite surgery case since the surface is now a sphere instead of a torus.

The paper is organized as follows. Section 2 is to set up some notation and conventions, and introduce some basic lemmas. Section 3 discusses immersed essential disks in tangle spaces. It will be shown that any such disk must intersect the axis of the tangle at some minimal number of points, and the disks are embedded and standard in certain cases. In Section 4 we define immersed surfaces that are in essential position with respect to an essential embedded surface and prove its existence in manifolds with finite fundamental groups. Section 5 defines elementary surfaces and shows that if the surface $F$ coming from an immersed $\pi_{1}$-injective torus is elementary, then the surgered manifold is either toroidal or the connected sum of two lens spaces. This is crucial in dealing with the fact that some of the knots excluded in Theorems 1.1 and 8.2 admit toroidal surgeries and hence the surgered manifold does contain $\pi_{1}$-injective tori. In Section 6 we define intersection graphs and prove some basic properties of such graphs. Section 7 defines angled Euler numbers and show that it is additive. Section 8 completes the proof of the main theorems.

The author would like to thank the referee for careful reading and some helpful comments. 


\section{Preliminaries}

In this paper we will consider both embedded surfaces and nonembedded surfaces in 3-manifolds. We always assume that surfaces and curves intersect transversely. Unless otherwise stated, surfaces $F$ in a 3-manifold $M$ are assumed to have boundaries on the boundary of $M$, and a homotopy of $F$ refers to a relative homotopy of the pair $(F, \partial F)$ in $(M, \partial M)$, i.e., a homotopy $f_{t}: F \rightarrow M$ such that $f_{t}(\partial F) \subset \partial M$ for all $t$. Similarly for arcs on surfaces.

An $\operatorname{arc} \alpha$ on a surface $F$ is trivial if it is rel $\partial \alpha$ homotopic to an $\operatorname{arc}$ on $\partial F$. If $\alpha$ is closed, then it is trivial if and only if it is null homotopic on $F$. A (possibly nonembedded) disk $D$ in a 3-manifold $M$ is nontrivial if it is not rel $\partial D$ homotopic to a disk on $\partial M$. If $M$ is irreducible (in particular if $M$ is the tangle space $E(t)$ below), then $D$ in $M$ is nontrivial if and only if $\partial D$ is a nontrivial curve on $\partial M$. A curve or disk is essential if it is nontrivial. Two (possibly immersed) curves $C_{1}, C_{2}$ on a surface $F$ intersect minimally if there is no subarc $a_{i} \subset C_{i}$ such that $\partial a_{1}=\partial a_{2}$ and the loop $a_{1} \cup a_{2}$ is null homotopic on $F$. When $C_{1}, C_{2}$ are embedded, this is equivalent to saying that $C_{1} \cup C_{2}$ contains no bigons on $F$, i.e., there are no arcs $a_{i} \subset C_{i}$ with $\partial a_{1}=\partial a_{2}$ such that $a_{1} \cup a_{2}$ bounds a disk on $F$ with interior disjoint from $C_{1} \cup C_{2}$.

In this paper a tangle is a triple $T=(B, t, m)$, where $B$ is a fixed 3-ball, $t=t_{1} \cup t_{2}$ is a pair of arcs properly embedded in $B$, and $m$ is a simple loop on $\partial B$, cutting $\partial B$ into two disks, called the left disk and the right disk, each containing two points of $\partial t$. The curve $m$ is called the axis of the tangle. Two tangles $(B, t, m)$ and $\left(B^{\prime}, t^{\prime}, m^{\prime}\right)$ are equivalent if they are homeomorphic as a triple. They are strongly equivalent if $B=B^{\prime}$ and the homeomorphism is the identity on $\partial B$.

Denote by $N(t)$ a regular neighborhood of $t$, and by $E(t)=B-\operatorname{Int} N(t)$ the exterior of $t$, which will also be called the tangle space of $(B, t, m)$. Denote by $A(t)$ the two annuli $A(t)=\partial N(t) \cap E(t)=A_{1}(t) \cup A_{2}(t)$ on $\partial E(t)$, by $P(t)$ the 4-punctured sphere $\partial B \cap E(t)$, and by $P_{1}(t) \cup P_{2}(t)$ the two twice-punctured disks obtained by cutting $P(t)$ along $m$. If $C$ is a properly embedded $(n-1)$ dimensional submanifold in an $n$-manifold $F$, denote by $F \mid C$ the manifold obtained by cutting $F$ along $C$.

Definition 2.1. A homotopy or isotopy $h_{x}$ of $E(t)$ or $\partial E(t)$ is P-preserving if $h_{x}$ maps each of the set $A(t), P_{1}(t), P_{2}(t), m$ to itself during the homotopy. Similarly, if $C$ is a curve or surface in $E(t)$, then a $P$-preserving homotopy or isotopy $h_{x}$ of $C$ is such that $C \cap Y$ is mapped to $Y$ for $Y=A(t), P_{1}(t), P_{2}(t), m$ and all $x \in[0,1]$.

A tangle $T=(B, t, m)$ is a $p / q$ rational tangle, denoted by $T(p / q)$, if $B$ is isotopic to a pillowcase with the four points of $\partial t$ as the cone points and $m$ a vertical circle, and $t$ is rel $\partial t$ isotopic to a pair of $\operatorname{arcs}$ on $\partial B$ of slope $p / q$. See [HT]. By definition $T(p / q)$ is equivalent to $T\left(p^{\prime} / q^{\prime}\right)$ if $p / q \equiv p^{\prime} / q^{\prime} \bmod 1$. The tangle is trivial if $q=0$ or 1 . We will always assume $q \geq 2$ and hence $T(p / q)$ is nontrivial. Denote by $E(p / q)$ the tangle space $E(t)$ if $(B, t, m)=T(p / q)$.

Definition 2.2. We use $\bar{p}=\bar{p}(p, q)$ to denote the $\bmod q$ inverse of $-p$ with minimal absolute value, i.e., $\bar{p}$ satisfies $p \bar{p} \equiv-1 \bmod q$, and $2|\bar{p}| \leq q$. Similarly, $\bar{p}_{i}$ denotes $\bar{p}\left(p_{i}, q_{i}\right)$ throughout the paper.

By a deformation of $B$ one can see that $T(p / q)=(B, t, m)$ can be isotoped so that $t$ is rel $\partial$ isotopic to a pair of vertical arcs, and $m$ is a curve of slope $\bar{p} / q$ 


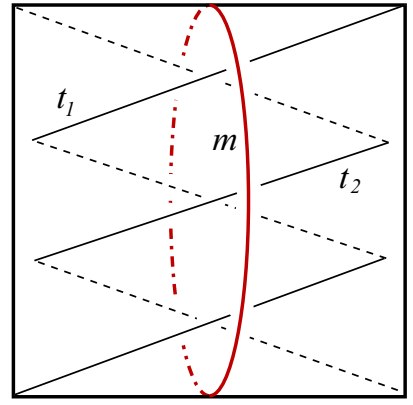

(a)

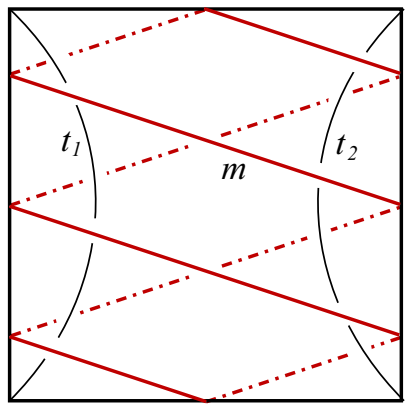

(b)

FIGURE 2.1

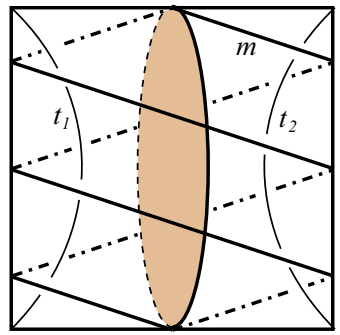

(a)

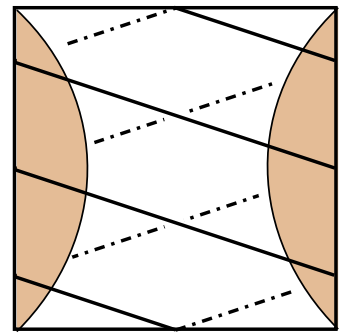

(b)

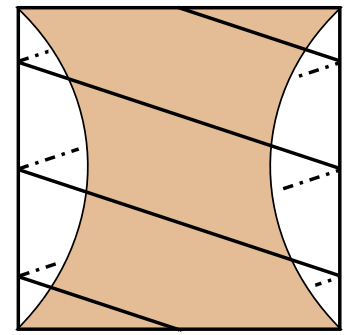

(c)

FIGURE 2.2

on $\partial B$. See Figure 2.1, where (a) is the standard picture of a $T(1 / 3)$, and (b) is $T(1 / 3)$ after the deformation. We have $\bar{p}=-1$ in this case, so $m$ is a curve of slope $-1 / 3$ on $\partial B$. In general this can be proved by lifting the boundary map to the universal cover of $\partial B$, considered as a sphere with four cone points of order 2 . It is represented by a matrix $A$ with $\operatorname{det}(A)=1$, which maps a line of slope $p / q$ to $1 / 0$. One can show that $A$ maps the line of slope $1 / 0$ to $\bar{p} / q$. We will use both points of view for $T(p / q)$ in what follows.

Let $E_{0}$ be an embedded disk in $B$ that separates the two arcs of $t$ and intersects $m$ in $2 q$ points; see Figure 2.2(a). Let $E_{i}(i=1,2)$ be embedded disks with $\partial E_{i}=t_{i} \cup \alpha_{i}$, where $\alpha_{i}$ is an arc on $\partial B$ intersecting $m$ at $q$ points; see Figure 2.2(b). These are chosen to be disjoint from each other. Now let $E_{3}$ be the disk in Figure $2.2(\mathrm{c})$, which intersects $E_{0}$ in one arc but has interior disjoint from $E_{1}$ and $E_{2}$, and we have $\partial E_{3}=t_{1} \cup \beta_{1} \cup t_{2} \cup \beta_{2}$, where $\beta_{1}, \beta_{2}$ are arcs on $\partial B$, each intersecting $m$ at $|\bar{p}|$ points. Thus $\left|\partial E_{3} \cap m\right|=2|\bar{p}|$. Note that $E_{3}$ is unique up to isotopy when $q>2$, and there are two such $E_{3}$ when $p / q=1 / 2$.

We call $E_{0}, E_{1}, E_{2}, E_{3}$ the standard disks for $(B, t)$. We will also use the same notation $E_{i}$ to denote the disk $E_{i} \cap E(t)$ when dealing with disks in $E(t)$.

An embedded disk $D$ in $E(t)$ is tight if it intersects $m$ and $A(t)$ minimally up to isotopy. $D$ is an $(r, s)$ disk if $|\partial D \cap A(t)|=r$ and $|\partial D \cap m|=s$. See Definition 3.1 for general definitions of tight curves and tight immersed disks. We need results to show that certain immersed disks are standard. As a warm up, we have the 
following lemma, which says that the standard disks are the standard models of tight $(r, s)$ disks in $E(t)$ when $r \leq 1$, or $r=2$ and $s$ is minimal. The proof is a standard innermost circle outermost arc argument by considering the intersection of $D$ with $E_{1} \cup E_{2}$, and is omitted. Certain versions of (2) and (3) are also true for immersed disks when changing isotopy to homotopy; see Lemmas 3.5 and 3.6.

Lemma 2.3. Suppose $(B, t, m)$ is a $p / q$ rational tangle. Let $\bar{p}$ be as in Definition 2.2 .

(1) If $D$ is an embedded tight $(0, s)$ disk in $E(t)$, then $s=2 q$ and $D$ is $P$-isotopic to the standard disk $E_{0}$.

(2) If $D$ is an embedded tight $(1, s)$ disk in $E(t)$, then $s=q$ and $D$ is $P$-isotopic to the standard disk $E_{1}$ or $E_{2}$.

(3) If $D$ is an embedded tight $(2, s)$ disk, then $s \geq 2|\bar{p}|$, and if $s=2|\bar{p}|$, then $D$ is $P$-isotopic to a standard disk $E_{3}$.

(4) Standard disks of type $E_{i}$ are unique up to isotopy unless $q=2$ and $i=3$, in which case there are exactly two such isotopy classes.

Denote by $|X|$ the number of components of $X$. Note that if $X$ is an immersed curve or surface in a manifold $M$, then it may have intersection between different components, in which case $|X|$ denotes the number of components before immersion, not the number of the components of its image in $M$. Thus two components of an immersed surface which intersect in $M$ are still considered as different components when counting $|X|$.

\section{IMMERSED DISKS IN TANGLE SPACES}

Definition 3.1. (1) A (possibly nonsimple) closed curve $C$ on $\partial E(t)$ is a tight curve if each component of $C \cap A(t), C \cap P_{1}(t)$ and $C \cap P_{2}(t)$ is nontrivial.

(2) A (possibly nonclosed) curve $C$ is an $(r, s)$-curve (or a curve of type $(r, s)$ ) if $|C \cap A(t)|=r$, and $|C \cap m|=s$.

(3) A disk $D$ in $E(t)$ is an $(r, s)$-disk (or a disk of type $(r, s))$ if $\partial D \cap \partial E(t)$ is a $(r, s)$-curve on $\partial E(t)$. It is a tight disk if $\partial D \subset \partial E(t)$ is tight.

Note that if $C$ is a tight curve on $\partial E(t)$, then each arc component of $C \cap A(t)$ is homotopic to an embedded arc. However, $C \cap P_{i}(t)$ may contain arcs which have self-intersections that cannot be removed by homotopy.

Lemma 3.2. (1) Suppose $C$ is a tight curve on $F=\partial E(t)$. Then it has minimal intersection with both $m$ and $A(t)$ up to homotopy.

(2) Any curve $C$ on $F$ is homotopic to a tight curve $C^{\prime}$, which is unique up to P-homotopy. In particular, if two tight curves are homotopic, then they are P-homotopic.

(3) Suppose $C, C^{\prime}$ are tight curves on $\partial E(t)$. If there are arcs $\alpha \subset C$ and $\beta \subset C^{\prime}$ such that $\partial \alpha=\partial \beta$ and $\alpha \cup \beta$ is a trivial loop on $\partial E(t)$, then $|\alpha \cap m|=|\beta \cap m|$ and $|\alpha \cap A(t)|=|\beta \cap A(t)|$.

Proof. (1) If $C$ is homotopic to $C_{1}$ which has fewer intersections with $m$, say, then the homotopy is a map $\varphi$ from an annulus $H=S^{1} \times I$ to $\partial E(t)$. Since $m \cup \partial A(t)$ is embedded, by transversality $\gamma=\varphi^{-1}(m \cup \partial A(t))$ is an embedded 1-manifold on $H$. By a homotopy we may assume $\gamma$ has no trivial loops. Since $|C \cap m|>\left|C_{1} \cap m\right|, \gamma$ contains a trivial arc on $H$ with both endpoints on $C$. An outermost such arc in $\gamma$ then cuts off a disk $D$ which gives rise to a homotopy from an $\operatorname{arc} \alpha$ of $C$ to an $\operatorname{arc}$ 
on $m$ or $\partial A(t)$, and the image of the interior of $D$ is disjoint from $m \cup \partial A(t)$ and hence is mapped into some $P_{i}(t)$ or $A(t)$. It follows that $\alpha$ is a trivial arc on $P_{i}(t)$ or $A(t)$, contradicting the assumption that $C$ is tight.

(2) It is clear that any curve is homotopic to a tight curve. We only need to prove the uniqueness. Let $\varphi: H \rightarrow \partial E(t)$ be a homotopy from $C_{1}$ to $C_{2}$ and assume $C_{i}$ are tight. Consider $\gamma=\varphi^{-1}(m \cup \partial A(t))$. As in the proof of (1) we may assume $\varphi$ has no trivial loops, and the tightness of $C_{i}$ implies that there are no trivial arcs, hence each component of $\gamma$ is an essential arc. Deform $\varphi$ so that $\gamma$ is a product $X \times I \subset S^{1} \times I=H$, where $X$ is a finite set in $S^{1}$. Clearly $\varphi$ is now a $P$-homotopy since it maps each $z \times I$ to an arc in some $P_{j}(t)$ or $A(t)$.

(3) Let $\varphi: D \rightarrow \partial E(t)$ be a map with $\partial D$ mapped to $\alpha \cup \beta$, chosen to have minimal intersection with $m \cup \partial A(t)$. Then $\varphi^{-1}(m \cup \partial A(t))$ has no loops, and each arc must have one end on $\alpha$ and the other on $\beta$ as otherwise we can get a contradiction as above. It follows that $|\alpha \cap \gamma|=|\beta \cap \gamma|$ for each component $\gamma$ of $m \cup \partial A(t)$.

Lemma 3.3. Suppose that $(B, t, m)$ is a $p / q$ rational tangle with $q \geq 2$, and $D$ is a nontrivial $(0, s)$ disk in $E(t)$. Then $s \geq 2 q$.

Proof. Let $E_{0}$ be the standard disk separating $t_{1}, t_{2}$, as defined in Section 2. Then $E_{0}$ cuts the tangle space $E(T)$ into two solid tori $V_{1}, V_{2}$, and $\partial E_{0}$ cuts the 4punctured disk $\partial B \cap E(t)$ into two twice-punctured disks $Q_{1}, Q_{2}$. We may assume that $D$ is tight, and $D \cap E_{0}$ consists of arcs, each of which is embedded in $E_{0}$.

Assume to the contrary that $s<2 q$. Among all such disks, choose $D$ so that $k=\left|\partial D \cap \partial E_{0}\right|$ is minimal. First assume $k>0$, and let $\alpha$ be an arc component of $D \cap E_{0}$, which cuts $E_{0}$ into $D_{1}^{\prime}$ and $D_{2}^{\prime}$. Without loss of generality we may assume that $\left|D_{1}^{\prime} \cap m\right| \leq q$. Cutting $D$ along $\alpha$ and pasting two copies of $D_{1}^{\prime}$, we obtain two disks $D_{1}, D_{2}$. Since $\left|\partial D_{1} \cap m\right|+\left|\partial D_{2} \cap m\right|=|\partial D \cap m|+2\left|\partial D_{1}^{\prime} \cap m\right|<4 q$, one of the $D_{i}$, say $D_{1}$, has $\left|\partial D_{1} \cap m\right|<2 q$. Since $D_{1}$ can be perturbed to have fewer intersections with $E_{0}$, by our choice of $D$ the curve $\partial D_{1}$ must be trivial. Write $\partial D_{1}=\alpha \cup \beta$ with $\alpha \subset \partial D$ and $\beta \subset \partial E_{0}$. Since both $D$ and $E_{0}$ are tight, by Lemma 3.2(3) we have $|\alpha \cap m|=|\beta \cap m|$. We can now homotope $D$ so that $\alpha$ is deformed to $\beta$ and then push off $E_{0}$. This reduces $\left|\partial D \cap \partial E_{0}\right|$ without changing $|\partial D \cap m|$, contradicting the choice of $D$.

We now assume that $\partial D \cap \partial E_{0}=\emptyset$, so $D \subset V_{1}$, say. Note that $m$ intersects $Q_{1}$ in $q$ arcs $\alpha_{1}, \ldots, \alpha_{q}$, each cutting $Q_{1}$ into a surface $F_{i} \subset Q_{1}$ which is the union of two longitudinal annuli. If $\partial D$ is disjoint from some $\alpha_{i}$, then $\partial D \subset F_{i}$, so $\partial D$ would be null homotopic on $F_{i}$ because $F_{i}$ is longitudinal while $\partial D$ is null homotopic in $V_{1}$, which contradicts the assumption that $D$ is nontrivial. Hence $\left|\partial D \cap \alpha_{i}\right|>0$. Let $\beta_{i}$ be an arc on $E_{0}$ with $\partial \beta_{i}=\partial \alpha_{i}$. If $\left|\partial D \cap \alpha_{i}\right|=1$, then we would have two closed curves $\partial D$ and $\alpha_{i} \cup \beta_{i}$ on the annulus $Q_{1} \cup E_{0}$ intersecting transversely at a single point, which is absurd. Therefore $\left|\partial D \cap \alpha_{i}\right| \geq 2$ for each $i$, hence $s=|\partial D \cap m| \geq 2 q$.

Lemma 3.4. Suppose that $(B, t, m)$ is a $p / q$ rational tangle. Let $\bar{p}$ be as in Definition 2.2. If $D$ is a nontrivial $(r, s)$ disk in $E(t)$, then $|s| \geq q$ if $r$ is odd, and $s \geq 2|\bar{p}|$ if $r$ is even.

Proof. We may assume that $D$ is tight. Consider the standard disks $E_{1}, E_{2}$ defined in Section 2. Let $E=E_{1} \cup E_{2}$. We proceed by induction on $(r,|D \cap E|)$. By Lemma 


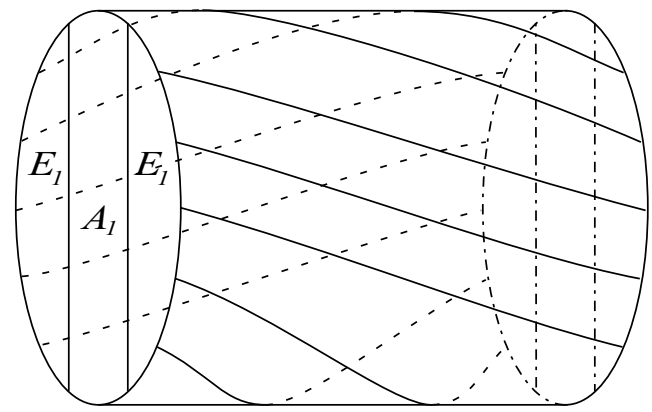

FiguRE 3.1

3.3 the result is true if $r=0$. Cutting $E(t)$ along $E$ produces a 3-ball on which $P(t)$ becomes an annulus $F$ and $m$ becomes a set of $2 q$ essential arcs on $F$. See Figure 3.1 for the case where $q=5$ and $\bar{p}=2$. Each boundary component $\partial_{i}$ of $F$ consists of four arcs, $\partial_{i}=a_{i}^{\prime} \cup b_{i}^{\prime} \cup a_{i}^{\prime \prime} \cup b_{i}^{\prime \prime}$, where $a_{i}^{\prime}, a_{i}^{\prime \prime}$ are from $\partial A_{i}(t)$ and $b_{i}^{\prime}, b_{i}^{\prime \prime}$ are copies of $\partial E_{i} \cap P(t)$. From Figure 3.1 it is easy to see that the condition $2|\bar{p}| \leq q$ in the definition of $\bar{p}$ implies that any arc $\gamma$ with endpoints on $a_{1}^{\prime} \cup a_{1}^{\prime \prime} \cup a_{2}^{\prime} \cup a_{2}^{\prime \prime}$ either is rel $\partial$ homotopic to an arc on one of the $a_{i}^{\prime}$ or $a_{i}^{\prime \prime}$ and hence is trivial on $P(t)$, or it intersects $m$ at least $|\bar{p}|$ times. Moreover, if $\gamma$ has both endpoints on the same boundary component of $F$, then it intersects $m$ at least $q$ times. If $r$ is odd, then there is at least one arc with both endpoints on the same component of $\partial F$, which intersects $m$ at least $q$ times; if $r$ is even, then there are at least two arcs, so they intersect $m$ at least $2|\bar{p}|$ times. Hence the result is true if $|D \cap E|=0$.

Now suppose $|D \cap E|>0$ and let $\alpha$ be an arc component of $D \cap E_{i}$. Then $\alpha$ cuts $D$ into $D^{\prime}, D^{\prime \prime}$ and it cuts $E_{i}$ into $E_{i}^{\prime}, E_{i}^{\prime \prime}$, with $E_{i}^{\prime \prime}$ the one containing the arc on $A(t)$. The four disks $D_{1}=D^{\prime} \cup E_{i}^{\prime}, D_{2}=D^{\prime} \cup E_{i}^{\prime \prime}, D_{3}=D^{\prime \prime} \cup E_{i}^{\prime}$, and $D_{4}=D^{\prime \prime} \cup E_{i}^{\prime \prime}$ are nontrivial. For if $D_{1}$ is trivial, say, then we can homotope $D^{\prime}$ to $E_{i}^{\prime}$ and then push off $E$ to reduce $|D \cap E|$; by Lemma 3.2(3) this will not change $|D \cap m|$ and $|D \cap A(t)|$ and hence is a contradiction to the minimality of $|D \cap E|$.

Suppose $D^{\prime}, D^{\prime \prime}, E_{i}^{\prime}, E_{i}^{\prime \prime}$ are of types $\left(r_{1}, s_{1}\right),\left(r_{2}, s_{2}\right),\left(0, s_{3}\right),\left(1, s_{4}\right)$, respectively. Then $r=r_{1}+r_{2}, s=s_{1}+s_{2}$, and $s_{3}+s_{4}=q$. The type of each $D_{i}$ is the sum of the types of the corresponding subdisks, so the four disks $D_{i}$ are of the following types:

$D_{1}: \quad\left(r_{1}, s_{1}+s_{3}\right)$,

$D_{2}: \quad\left(r_{1}+1, s_{1}+s_{4}\right)$,

$D_{3}: \quad\left(r_{2}, s_{2}+s_{3}\right)$,

$D_{4}: \quad\left(r_{2}+1, s_{2}+s_{4}\right)$.

First assume $r$ is odd. Then we may assume without loss of generality that $r_{1}$ and $r_{2}+1$ are odd. This implies that $r_{1}, r_{2}+1 \leq r$. Apply induction to $D_{1}$ and $D_{4}$ (note that they can be deformed to have fewer intersections with $E$ ), we have $s_{1}+s_{3} \geq q, s_{2}+s_{4} \geq q$. Adding these together gives $s+q=s_{1}+s_{2}+s_{3}+s_{4} \geq 2 q$, hence $s \geq q$.

Now assume $r$ is even. If $r_{1}, r_{2}$ are odd, then $r_{i}+1 \leq r$, so by induction we have $s_{1}+s_{3} \geq q, s_{1}+s_{4} \geq 2|\bar{p}|, s_{2}+s_{3} \geq q$, and $s_{2}+s_{4} \geq 2|\bar{p}|$. Adding these together gives $2 s+2 q=2\left(s_{1}+s_{2}+s_{3}+s_{4}\right) \geq 2 q+4|\bar{p}|$, hence $s \geq 2|\bar{p}|$. If both $r_{i}$ are even and nonzero, then a similar argument as above applies. So we now assume that $r_{1}=0$ 
and $r_{2}=r \geq 2$. Then we can apply induction to $D_{2}$ and $D_{3}$ to get $s_{1}+s_{4} \geq q$ and $s_{2}+s_{3} \geq 2|\bar{p}|$. Adding these together gives $q+s=s_{1}+s_{2}+s_{3}+s_{4} \geq q+2|\bar{p}|$, hence $s \geq 2|\bar{p}|$, as required.

Suppose $F$ is an immersed surface represented by $\varphi: \tilde{F} \rightarrow M$. Let $S$ be an embedded surface in $M$. Then $C=\varphi^{-1}(S)$ is an embedded 1-manifold on $\tilde{F}$, and $\varphi(C)$ is an immersed 1-manifold on $S$. As in the embedded case, we use $C=F \cap S$ to denote both the 1-manifold $C=\varphi^{-1}(S)$ on $\tilde{F}$ and the immersed curve $\varphi: C \rightarrow S$ on $S$. To simplify notation, we will not distinguish $\tilde{F}$ and $F$ and simply refer $C \subset \tilde{F}$ as $C \subset F$. Thus the curve $C$ above is still considered embedded on $F$ even though it may have self-intersection when considering $F$ as a subset of $M$.

The following two lemmas show that immersed tight $(1, q)$ disks and $(2,2|\bar{p}|)$ disks are standard up to $P$-homotopy.

Lemma 3.5. Suppose $D$ is an immersed tight $(2,2|\bar{p}|)$ disk in $E(p / q)$. Then it is $P$-homotopic to a standard disk $E_{3}$ defined in Section 2.

Proof. By Lemma 3.2(2), if two tight curves are homotopic, then they are $P$ homotopic; hence we need only show that $D$ is homotopic to some $E_{3}$.

If $|D \cap E|=0$, then $D$ lies on the 3-ball shown in Figure 3.1, with $\partial D$ consisting of one arc on each $A_{i}(t)$ and two $\operatorname{arcs} c_{1}, c_{2}$ on the annulus $F$ obtained by cutting $P(t)$ along $E$. From Figure 3.1 we can see that the minimal intersection number between $m$ and a nontrivial arc on $F$ is $|\bar{p}|$, and there are exactly two such arcs if $q>2$, or four such arcs if $q=2$. These are on the boundary of the standard disks of type $E_{3}$. The assumption that $D$ is a $(2,2|\bar{p}|)$ disk implies that $\left|c_{i} \cap m\right|=|\bar{p}|$. Hence we can deform $c_{i}$ by a homotopy of $(F, m)$ so that $\partial D$ matches the boundary of a standard disk $E_{3}$. Since $E(t)$ is irreducible, a further $P$-homotopy deforms $D$ to $E_{3}$.

Now assume $|D \cap E|>0$. Examine the proof of Lemma 3.4. If one of $\partial D_{i}$ $(i=1,2,3,4)$ is trivial, then we may reduce $|D \cap E|$ by $P$-homotopy and the result follows by induction, so we may assume that they are all nontrivial.

We may assume $s_{1} \leq s_{2}$, so $s_{1}+s_{2}=2|\bar{p}| \leq q$ implies $s_{1}<q$. Recall that $s_{3}+s_{4}=q$. By the proof of Lemma 3.4, if $r_{1}=0$, then $D_{1}$ would be a $\left(0, s_{1}+s_{3}\right)$ disk, but since $s_{1}<q$ and $s_{3} \leq q$, we would have $s_{1}+s_{3}<2 q$, contradicting Lemma 3.3. Therefore $r_{1}=r_{2}=1$. Since $D_{1}$ is a $\left(1, s_{1}+s_{3}\right)$ disk, by Lemma 3.4 we have $s_{1}+s_{3} \geq q$, so $s_{3}+s_{4}=q$ implies $s_{1} \geq s_{4}$. Similarly we have $s_{2} \geq s_{4}$. Since $D_{2}$ is of type $\left(2, s_{1}+s_{4}\right)$, we have $2|\bar{p}|=s_{1}+s_{2} \geq s_{1}+s_{4} \geq 2|\bar{p}|$, where the last inequality follows from Lemma 3.4. Hence we must have $s_{2}=s_{4}$. By symmetry we have $s_{1}=s_{4}$. Thus both $D_{2}$ and $D_{4}$ are $(2,2|\bar{p}|)$ disks, so by induction they are $P$-homotopic to $E_{3}$. On the other hand, by construction one of the $D_{2}, D_{4}$ has the property that it has both arcs of $D_{i} \cap A(t)$ on the same component of $A(t)$, which is a contradiction because $E_{3}$ has one edge on each of $A_{1}(t)$ and $A_{2}(t)$.

Lemma 3.6. Suppose $D$ is a tight $(1, q)$ disk. Then it is P-homotopic to the standard disk $E_{1}$ or $E_{2}$.

Proof. Let $D_{1}, \ldots, D_{4}$ and $r_{i}, s_{i}$ be as defined in the proof of Lemma 3.4. The result is clear when $|D \cap E|=0$, and it follows by induction if one of the $D_{i}$ is trivial, so assume $|D \cap E|>0$ and $\partial D_{i}$ is nontrivial for $i=1,2,3,4$. Since $r=r_{1}+r_{2}=1$, we may assume $r_{1}=0$. The disk $D_{1}$ is of type $\left(0, s_{1}+s_{3}\right)$, so by Lemma 3.3 we 
must have $s_{1}+s_{3} \geq 2 q$. On the other hand, we have $s_{1}+s_{2}=s_{3}+s_{4}=q$, so $s_{2}=s_{4}=0$. Now $D_{4}$ is a $(2,0)$ disk, contradicting Lemma 3.4 .

We note that a statement similar to Lemmas 3.5 and 3.6 is not true for $(0,2 q)$ disks. A nonstandard $(0,2 q)$ disk can be formed by winding $E_{0}$ around $A_{i}(t)$. In other words, we can take $E_{0}$ and $n$ copies of $A_{1}$ or $A_{2}$ (but not both) and then cut and paste to form a disk which is still an immersed $(0,2 q)$ disk. We call such a surface an $n$-winding disk if $n$ is the minimal number of tubes required in the above construction. The curve $m$ cuts the boundary of a $(0,2 q)$ disk $D$ into $2 q$ arcs. We leave it to the reader to verify that if $D$ is an $n$-winding disk, then two of those $2 q$ arcs must have self-intersection if $n>0$.

Lemma 3.7. Suppose $D$ is a tight $(0,2 q)$ disk in $E(p / q)$. Then $D$ is P-homotopic to an $n$-winding disk for some $n$. In particular, if each component of $\partial D \cap P(t)$ is an embedded arc, then $D$ is $P$-homotopic to $E_{0}$.

Proof. First assume $D \cap E=\emptyset$. Cutting $E(t)$ along $E$ produces a $D^{2} \times I$, where $P(t)$ becomes an annulus $F$ and $m$ becomes a set of $2 q$ parallel essential arcs on $F$, so it cuts $F$ into $2 q$ squares; see Figure 3.1. Since $|\partial D \cap m|=2 q$ and $D$ is tight, $m$ cuts $\partial D$ into $2 q$ arcs, each of which is nontrivial and hence connects one component of $m$ on $F$ to another. It follows that each square contains exactly one arc of $\partial D$, which can be straightened inside of the square. Therefore $\partial D$ is homotopic to the core of $F$, and $D$ is homotopic to $E_{0}$. By Lemma 3.2(2) $D$ is $P$-homotopic to $E_{0}$.

Now assume $|D \cap E|>0$. Consider an arc $\alpha \subset D \cap E$ which is outermost on $D$. Let $D^{\prime}, D^{\prime \prime}, E^{\prime}, E^{\prime \prime}$ and $D_{1}, \ldots, D_{4}$ be as in the proof of Lemma 3.4, with $D^{\prime}$ an outermost disk on $D$. Then $D_{1}$ is a $\left(0, s_{1}+s_{3}\right)$ disk and $D_{3}$ is a $\left(0, s_{2}+s_{3}\right)$ disk, so we have $s_{1}+s_{3} \geq 2 q$ and $s_{2}+s_{3} \geq 2 q$. Since $s_{1}+s_{2}=2 q$ and $s_{3} \leq s_{3}+s_{4}=q$, we must have $s_{1}=s_{2}=s_{3}=q$ and $s_{4}=0$. It follows that there are exactly two outermost disks on $D$, and all other components $Q$ of $D$ cut along $D \cap E$ are bigons in the sense that it intersects $E$ in exactly two arcs, and $Q \cap m=\emptyset$. The union of $Q$ with two disks on $E$ forms a $(2,0)$ disk, so by Lemma 3.4 it must be trivial. In particular all components of $D \cap E$ are on the same disk $E_{1}$, say. It is now easy to see that each $Q$ is a tube (i.e., an annulus parallel to $A_{1}(t)$ ) cut open, hence $D$ is an $n$-winding disk. If $n>0$, then by the above some component of $\partial D \cap P(t)$ must have self-intersection. Therefore if each component of $\partial D \cap P(t)$ is embedded, then we must have $n=0$, and hence $D$ is homotopic to $E_{0}$.

\section{IMMERSED SURFACES IN ESSENTIAL POSITION}

Recall that an embedded orientable surface $F$ in a 3 -manifold is essential if it is incompressible, $\partial$-incompressible, and no component of $F$ is boundary parallel.

Lemma 4.1. Let $F$ be an embedded orientable essential surface in a 3-manifold $M$. Then no immersed essential arc $\alpha$ on $F$ is rel $\partial \alpha$ homotopic to an arc on $\partial M$.

Proof. Suppose $\alpha$ is rel $\partial \alpha$ homotopic to an $\operatorname{arc} \beta$ on $\partial M$, and let $D$ be a nullhomotopy disk bounded by $\alpha \cup \beta$. If $M$ is reducible, then since $F$ is essential we may assume it is disjoint from a reducing sphere $S$, and we can then modify $D$ if necessary to make it disjoint from $S$. Therefore by decomposing along $S$ we may assume that $M$ is irreducible. Similarly, since $F$ is incompressible and $\partial$ incompressible and $M$ is irreducible, $F$ can be isotoped and $D$ can be modified to 
be disjoint from any $\partial$-reducing sphere, hence by cutting along $\partial$-reducing disks if necessary, we may also assume that $M$ is $\partial$-irreducible.

Let $2 M$ be the double of $M$ along $\partial M$, and let $2 F$ be the double of $F$ along $\partial F=F \cap \partial M$. Then the double of $\alpha$ is an essential curve on $2 F$ which is null homotopic in $2 M$. Thus $2 F$ is not $\pi_{1}$-injective and hence is compressible. Let $D$ be a compressing disk of $2 F$ in $2 M$ such that $|D \cap \partial M|$ is minimal. Since $F$ and $\partial M$ are incompressible in $M$, by an innermost circle argument one can show that $D \cap \partial M$ has no circle component, and we must have $D \cap \partial M \neq \emptyset$ because $D$ cannot be a compressing disk of $F$. An outermost arc of $D \cap \partial M$ then cuts off a $\partial$-compressing disk of $F$ in $M$.

Lemma 4.2. Let $F$ be an embedded orientable essential surface in a 3-manifold $M$. Suppose $\rho: \tilde{M} \rightarrow M$ is a finite cover. Then the surface $\tilde{F}=\rho^{-1}(F)$ is essential in $\tilde{M}$.

Proof. Let $\tilde{F}_{1}$ be a component of $\tilde{F}$, and let $F_{1}$ be the corresponding component in $F$. If $\tilde{F}_{1}$ is boundary parallel, then it cuts off a regular neighborhood of a boundary component $\tilde{T}$ of $\tilde{M}$, which projects to a regular neighborhood of a boundary component $T$ of $M$, hence $F_{1}$ would also be boundary parallel, contradicting the assumption that $F$ is essential.

A compressing disk of $\tilde{F}_{1}$ would map to an immersed disk in $M$ with boundary an essential curve on $F_{1}$, which contradicts the fact that an incompressible surface is $\pi_{1}$-injective. Therefore $\tilde{F}$ is incompressible in $\tilde{M}$.

If $\tilde{F}$ is $\partial$-compressible, then a $\partial$-compressing disk of $\tilde{F}$ in $\tilde{M}$ projects to a disk in $M$ whose boundary consists of an essential arc on $F$ and an arc on $\partial M$, which contradicts Lemma 4.1.

Definition 4.3. Suppose $L$ is a link in a 3 -manifold $M$ and $F$ is an embedded essential surface in $E(L)=M-\operatorname{Int} N(L)$ with nonempty nonmeridional boundary slope on each boundary component of $E(L)$. Let $\hat{S}$ be an immersed surface in $M$, and let $S=\hat{S} \cap E(L)$. Then $\hat{S}$ is said to be in essential position with respect to $F$ if $\hat{S} \cap L \neq \emptyset$, each arc component of $S \cap F$ is essential on both $S$ and $F$, and each circle component is nontrivial on $S$.

The following lemma is essentially due to Gabai [Ga].

Lemma 4.4 (Thin Position Lemma). Suppose $L$ is a link in $S^{3}$, and $F$ is an embedded essential surface in $E(L)=S^{3}-\operatorname{Int} N(L)$ with nonempty nonmeridional boundary slopes on each boundary component of $E(L)$. Then there exist an embedded sphere $\hat{S}$ in $S^{3}$ which is in essential position with respect to $F$.

Proof. When $L$ is a knot this is [Ga, Lemma 4.4]. If $L$ splits, one can use the incompressibility of $F$ to find a splitting sphere disjoint from $F$ and proceed by induction to find $\hat{S}$ on the side containing $F$; hence we may assume that $L$ is nonsplit. In this case the proof is the same as in [Ga, Lemma 4.4]. Put $L$ in thin position, and let $\hat{S}$ be a thin level surface. Since $L$ is nonsplit, $\hat{S}$ has nonempty intersection with $L$. Isotoping $F$ properly and using the argument in [Ga, Lemma 4.4], one can show that $\hat{S}$ can be chosen so that it has neither high disk nor low disk, in which case the arc components of $S \cap F$ are essential on both $F$ and $S$. Since $F$ is incompressible, any circle of $F \cap S$ which is trivial on $S$ bounds a disk on $F$, so one can modify $S$ 
by cut and paste to get rid of those curves. We refer the reader to the proof of Ga, Lemma 4.4] for details.

The following can be considered as an immersed version of the thin position lemma. It works for links in manifolds with finite fundamental group.

Lemma 4.5 (Immersed Thin Position Lemma). Suppose $M$ is a closed irreducible 3-manifold with finite fundamental group, $L$ a link in $M$, and $F$ an embedded essential surface in $E(L)=M-\operatorname{Int} N(L)$ with nonempty nonmeridional boundary slopes on each boundary component of $E(L)$. Then there exists an immersed sphere $Q^{\prime}$ in $M$ which is in essential position with respect to $F$.

Proof. The universal cover $\tilde{M}$ of $M$ is a simply connected closed 3-manifold and hence by the Poincaré conjecture proved by Perelman $[\mathrm{Pr}]$, it is an $S^{3}$. The lifting of $L$ is a link $\tilde{L}$ in $\tilde{M}$, and the lifting of $F$ is a surface $\tilde{F}$ in $E(\tilde{L})=S^{3}-\operatorname{Int} N(\tilde{L})$. Since $F$ is an essential embedded surface in $E(L)$, by Lemma $4.2 \tilde{F}$ is an essential embedded surface in $\tilde{M}=E(\tilde{L})$. By the Thin Position Lemma above, there is a sphere $\tilde{Q}^{\prime}$ in $S^{3}$ which is in essential position with respect to $\tilde{F}$. Let $Q^{\prime}$ be the projections of $\tilde{Q}^{\prime}$ in $M$, let $\tilde{Q}=\tilde{Q}^{\prime} \cap E(\tilde{L})$, and let $Q=Q^{\prime} \cap E(L)$. The preimage of an arc in $Q \cap F$ may consist of several arcs, but at least one of them is in $\tilde{Q} \cap \tilde{F}$, and an inessential arc would lift to an inessential arc. Therefore all arcs of $Q \cap F$ are essential on both $F$ and $Q$. Similarly all circle components of $Q \cap F$ are essential on $Q$.

\section{Elementary SURFACES}

We will always use $K_{r}$ to denote the core of the Dehn filling solid torus in the surgered manifold $K(r)$. Given an immersed surface $F$ in $E(K \cup C)$ with each boundary component either a meridional curve on $\partial N(C)$ or a curve of slope $r$ on $\partial N(K)$, denote by $\hat{F}$ the closed surface obtained by attaching a meridian disk of $N\left(K_{r} \cup C\right)$ to each boundary component of $F$.

Suppose $K=K\left(r_{1}, r_{2}, r_{3}\right)$ is a Montesinos knot of length 3 , where $r_{i}=p_{i} / q_{i}$ and $q_{i} \geq 2$. Let $L=K \cup C$, where $C$ is the axis of $K$ as shown in Figure 5.1. Let $E(L)=S^{3}-\operatorname{Int} N(L)$ be the exterior of $L$. Denote by $V$ the solid torus $S^{3}-\operatorname{Int} N(C)$. There are three disks $D_{1}, D_{2}, D_{3}$ cutting the pair $(V, K)$ into three rational tangles $\left(B_{i}, t_{i}, m_{i}\right)$ of slope $r_{i}$. Let $P_{i}$ be the twice punctured disk $D_{i} \cap E(L)$, and let $P=P_{1} \cup P_{2} \cup P_{3}$. Then $P=P_{1} \cup P_{2} \cup P_{3}$ cuts $E(K \cup C)$ into three tangle spaces $E\left(t_{1}\right), E\left(t_{2}\right), E\left(t_{3}\right)$. The surface $\partial E\left(t_{i}\right)$ is the union of $P_{i}, P_{i+1}$ and three annuli $A_{0}\left(t_{i}\right), A_{1}\left(t_{i}\right), A_{2}\left(t_{i}\right)$, where $A_{0}\left(t_{i}\right)=\partial E\left(t_{i}\right) \cap \partial N(C)$ and $A_{1}\left(t_{i}\right) \cup A_{2}\left(t_{i}\right)$ are the ones on $\partial N(K)$.

Definition 5.1. An immersed surface $F$ in $E(K \cup C)$ is an elementary surface if the following holds:

(1) each component of $F \cap E\left(t_{i}\right)$ is a tight disk of type $\left(0,2 q_{i}\right),\left(1, q_{i}\right)$ or $\left(2,2\left|\bar{p}_{i}\right|\right)$;

(2) at least one $F \cap E\left(t_{i}\right)$ has no disk of type $\left(0,2 q_{i}\right)$;

(3) if $q_{i}=2$, then all disks of type $\left(2,2\left|\bar{p}_{i}\right|\right)$ in $E\left(t_{i}\right)$ are homotopic to each other; and

(4) type $\left(0,2 q_{i}\right)$ and type $\left(2,2\left|\bar{p}_{i}\right|\right)$ disks do not appear simultaneously in any $E\left(t_{i}\right)$. 
Lemma 5.2. Suppose $K$ is hyperbolic and there is an embedded closed torus or Klein bottle $\hat{F}$ in $K(r)$ such that $F=\hat{F} \cap E(K \cup C)$ is an elementary surface. Then $K(r)$ is toroidal.

Proof. By considering the boundary of a regular neighborhood of $F$ if necessary, we may assume $F$ is orientable and hence is a punctured torus. Since $F$ is embedded and $F \cap E\left(t_{i}\right)$ is the union of standard disks in $E\left(t_{i}\right)$ for all $i$, the surface $\hat{F}$ is a candidate surface as defined in [HO. Since $\hat{F}$ is a torus, by [Wu3. Lemma 7.1], the knot $K$ and the boundary slope $r$ of $\hat{F}$ are among those listed in Wu3, Theorem 1.1]. By Wu3, Theorem 1.2] $\hat{F}$ is incompressible and hence $K(r)$ is toroidal.

The main result of this section is Proposition 5.6, which shows that the above lemma is still true for immersed $\hat{F}$.

Lemma 5.3. Suppose $F$ is an immersed elementary surface in $E(K \cup C)$.

(1) For each $i, F$ can be deformed so that $F \cap E\left(t_{i}\right)$ is embedded.

(2) $F$ can be deformed so that $F \cap P$ is embedded.

Proof. By Lemmas 3.5-3.7 each component of $F \cap E\left(t_{i}\right)$ can be deformed to a standard disk or an $n$-winding disk. By condition (2) in Definition 5.1, up to relabeling we may assume that there is no disk of type $\left(0,2 q_{1}\right)$ and hence no $n$ winding disk in $E\left(t_{1}\right)$ for $n>0$. Since standard disks are embedded, each arc of $F \cap P_{1}$ and $F \cap P_{2}$ is embedded up to homotopy. Since $K$ is a knot, at most one of the $q_{j}$ is even, hence without loss of generality we may assume that $q_{2}$ is odd. It means that a strand of $t_{2}$ has one endpoint on each of $P_{2}$ and $P_{3}$. If $F \cap E\left(t_{2}\right)$ contains an $n$-winding disk $D$ for some $n>0$, then $D \cap P_{2}$ would be an arc which is not homotopic to an embedded arc, a contradiction. Therefore $E\left(t_{2}\right)$ has no such disk, which implies that each component of $F \cap E\left(t_{2}\right)$ is also homotopic to a standard disk. Similarly, since each arc in $F \cap\left(P_{1} \cup P_{3}\right)$ is homotopic to an embedded arc, there is no $n$-winding disk in $E\left(t_{3}\right)$ for any $n>0$. Therefore each component of $F \cap E\left(t_{j}\right)$ is homotopic to a standard disk for all $j$.

By definition, standard disks can be homotoped in $E\left(t_{j}\right)$ to be disjoint from each other except that

(i) a disk of type $E_{0}$ intersects a disk of type $E_{3}$ essentially, and

(ii) when $q_{j}=2$, there are two possible homotopy classes of type $E_{3}$ which intersect each other essentially.

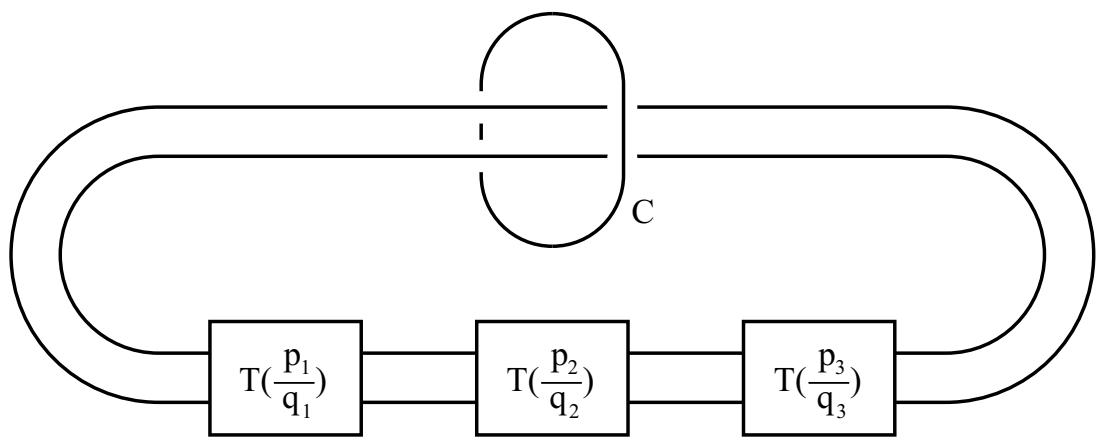

FiguRE 5.1 


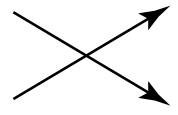

(a)

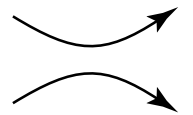

(b)

FiguRE 5.2

However, these have been excluded by conditions (3) and (4) in Definition 5.1. Therefore $F \cap E\left(t_{j}\right)$ can be deformed to be a set of mutually disjoint standard disks. This proves (1).

It may not be possible to do the above simultaneously for all the three tangles, but it implies that each $F \cap P_{i}$ can be deformed to be embedded, hence we can homotope $F$ so that $F \cap P$ is embedded, as stated in (2).

Lemma 5.4. Let $P$ be a twice-punctured disk, and $P_{0}, P_{1}$ the two boundary copies of $P$ in $P \times I$. Suppose $D_{1}$ is an embedded disk in $P \times I$ intersecting each $P_{i}$ at a single essential arc $a_{i}$. Let $D_{2}$ be an immersed disk in $P \times I$ with $b_{i}=D_{2} \cap P_{i}=a_{i}$ for $i=0,1$. Then $D_{2}$ is homotopic to $D_{1}$ rel $b_{0} \cup b_{1}$.

Proof. By an isotopy of $P \times I$ we may assume that $D_{1}$ is a product disk $\alpha \times I$. Let $\beta_{1}, \beta_{2}$ be two arcs on $P$ such that $\alpha, \beta_{1}, \beta_{2}$ are mutually disjoint, mutually nonparallel, and $\beta_{1} \cup \beta_{2}$ cuts $P$ into a disk. We may write $\partial D_{1}=a_{0} \cup a_{2} \cup a_{1} \cup a_{3}$ and $\partial D_{2}=b_{0} \cup b_{2} \cup b_{1} \cup b_{3}$, so that $\partial a_{2}=\partial b_{2}, \partial a_{3}=\partial b_{3}$, and $a_{2}, a_{3}, b_{2}, b_{3}$ are arcs on $\partial P \times I$. Put $Q_{i}=\beta_{i} \times I \subset P \times I$ and let $Q=Q_{1} \cup Q_{2}$. Homotope $D_{2}$ rel $b_{0} \cup b_{1}$ so that it has minimal intersection with $Q$. Let $\varphi: D \rightarrow D_{2}$ be the immersion. Then $\varphi^{-1}(Q)$ is an embedded 1-manifold on $D$. Using the fact that the $\operatorname{arc} \alpha, \beta_{1}, \beta_{2}$ is essential mutually nonhomotopic proper arcs in $P$ one can apply an innermost circle outermost arc argument to show that $D_{2} \cap Q=\emptyset$. Since $Q$ cuts $\partial P \times I$ into disks, we see that $a_{2} \cup b_{2}$ and $a_{3} \cup b_{3}$ are trivial loops, hence up to homotopy rel $b_{0} \cup b_{1}$ we can deform $\partial D_{2}$ to $\partial D_{1}$. Since $P \times I$ is irreducible, one can further deform $D_{2}$ to $D_{1}$ by a homotopy rel $\partial D_{2}$.

We now consider a set of oriented loops $C$ on a torus $T$ with oriented meridianlongitude pair $(\mu, \lambda)$. Then $C$ represents some $a \mu+b \lambda$ in $H_{1}(T)$. Define $b / a$ to be the slope of $C$. Note that $C$ is not required to be embedded. If $C$ has a crossing as in Figure 5.2(a), we can change it to that in Figure 5.2(b). This operation is called smoothing a crossing.

Lemma 5.5. Let $C$ be a set of oriented immersed curves on a torus $T$. Let $C^{\prime}$ be obtained from $C$ by smoothing crossings. Then $C$ and $C^{\prime}$ have the same slope on $T$.

Proof. This is obvious since the curves in Figure 5.2(a) and (b) represent the same homology class in $H_{1}(T)$.

Proposition 5.6. Suppose $K=K\left(p_{1} / q_{1}, p_{2} / q_{2}, p_{3} / q_{3}\right)$ is hyperbolic and $\hat{F}$ is an immersed $\pi_{1}$-injective torus in $K(r)$. If $F=\hat{F} \cap E(K \cup C)$ is an elementary surface, then $K(r)$ is toroidal.

Proof. As before, let $P=P_{1} \cup P_{2} \cup P_{3}$ be the three twice-punctured disks that cut $E(K \cup C)$ into $E\left(t_{1}\right) \cup E\left(t_{2}\right) \cup E\left(t_{3}\right)$. Let $W_{i}=P_{i} \times I$ be a collar of $P_{i}$. Then we can rewrite $E(K \cup C)$ as the union $\left(\bigcup W_{i}\right) \cup_{R}\left(\bigcup E\left(t_{i}\right)\right)$, where $W_{i}$ and $E\left(t_{j}\right)$ 


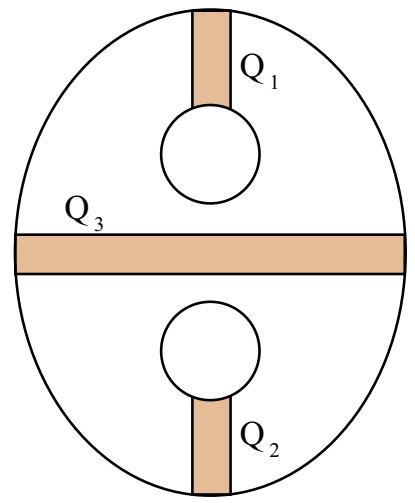

FiguRE 5.3

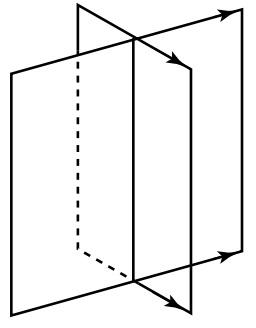

(a)

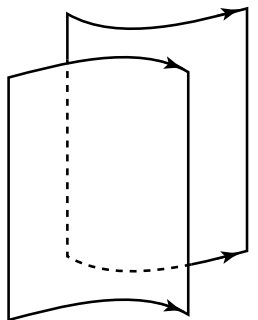

(b)

FiguRE 5.4

have mutually disjoint interiors, and $R=\left(\bigcup W_{i}\right) \cap\left(\bigcup E\left(t_{i}\right)\right)$ is the union of six twice-punctured disks.

Since $F$ is elementary, by Lemma $5.3(2)$ we may assume that $F \cap P$ is embedded, so $F \cap W_{i}$ consists of mutually disjoint product disks. Since the $E\left(t_{i}\right)$ are mutually disjoint, by Lemma 5.3(1), we can deform $F_{i}=F \cap E\left(t_{i}\right)$ in $E\left(t_{i}\right)$ so that it is embedded. Note that this can be done by a homotopy of the pair $\left(E\left(t_{i}\right), R \cap E\left(t_{i}\right)\right)$, so they can be combined and then extended into a small neighborhood of $R$ in $\bigcup W_{j}$ to form a homotopy of $F$. After this homotopy $F \cap W_{i}$ may no longer be embedded; however, since we started with product disks and the homotopy maps each component of $R$ to itself, each component of $F \cap W_{i}$ is still homotopic to a product disk.

Let $P_{i}^{\prime}, P_{i}^{\prime \prime}$ be the two copies of $P_{i}$ on $\partial W_{i}$. Since $F \cap E\left(t_{i}\right)$ is a union of mutually disjoint standard disks, we may assume that $F \cap P_{i}^{\prime}$ consists of embedded essential arcs in the small disks $Q_{1} \cup Q_{2} \cup Q_{3}$ shown in Figure 5.3. Since each component $D$ of $F \cap W_{i}$ is a disk, the two arcs $D \cap P_{i}^{\prime}$ and $D \cap P_{i}^{\prime \prime}$ must lie in $Q_{j} \times 0$ and $Q_{j} \times 1$ for the same $j$ because two essential arcs in different $Q_{j}$ are not homotopic to each other. Let $E$ be an embedded disk in $Q_{j} \times I$ such that $E \cap P_{i}^{\prime}=D \cap P_{i}^{\prime}$ and $E \cap P_{i}^{\prime \prime}=D \cap P_{i}^{\prime \prime}$. By Lemma $5.4 D$ is rel $D \cap\left(P_{i}^{\prime} \cup P_{i}^{\prime \prime}\right)$ homotopic to $E$. Therefore up to homotopy we may assume that each component $D$ of $F \cap W_{i}$ is a flat embedded disk in $Q_{j} \times I$, so different components $D^{\prime}, D^{\prime \prime}$ of $F \cap W_{i}$ are either disjoint or intersect at a single arc, as shown in Figure 5.4(a). 
Orient $\partial F$ so that all arcs of $\partial F \cap W_{i}$ run monotonically from $P_{i}^{\prime}$ to $P_{i}^{\prime \prime}$. We can then perform a double edge smoothing of $D^{\prime}, D^{\prime \prime}$ to obtain two new disks $D_{1}^{\prime}, D_{1}^{\prime \prime}$, as shown in Figure 5.4(b). After doing this for all double curves, the surface $F \cap W_{i}$ becomes a set of mutually disjoint embedded disks, and the original surface $F$ now becomes an embedded surface $F^{\prime}$ in $E(K \cup C)$. By definition $F^{\prime}$ is an elementary surface. Note that the double curve smoothing does not change the Euler characteristic of a surface. By Lemma $5.5 F^{\prime}$ has the same boundary slope as that of $F$. Since both $\partial F^{\prime}$ and $\partial F$ are embedded, we see that they have the same number of boundary curves. Let $\hat{F}^{\prime}$ be the closed surface obtained by adding meridian disks of $N\left(K_{r} \cup C\right)$ to $\partial F^{\prime}$. The above implies that $\chi\left(\hat{F}^{\prime}\right)=\chi(\hat{F})=0$, hence $\hat{F}^{\prime}$ is a torus or Klein bottle, and it is embedded. It now follows from Lemma 5.2 that $K(r)$ is toroidal.

\section{INTERSECTION GRAPHS}

We continue using the notation in Section 5 , so $K=K\left(p_{1} / q_{1}, p_{2} / q_{2}, p_{3} / q_{3}\right)$, $L=K \cup C$, and $P=P_{1} \cup P_{2} \cup P_{3}$, cutting $E(L)$ into three tangle spaces $E\left(t_{1}\right), E\left(t_{2}\right)$, $E\left(t_{3}\right)$. Let $K(r)$ be the manifold obtained by Dehn surgery along a slope $r$ on $K$. Denote by $K_{r}$ the core of the Dehn filling solid torus in $K(r)$, and let $L_{r}=K_{r} \cup C$.

Lemma 6.1. $P$ is an essential surface in $E(L)$.

Proof. If $P$ is compressible, then the compressing disk would separate the two strings of $t$ and $t$ would be a trivial tangle, contradicting the assumption that $q_{i} \geq 2$. It is well known that if $\partial M$ is a set of tori, then any connected incompressible surface in $M$ is also $\partial$-incompressible unless it is an annulus. Hence $P$ is also $\partial$-incompressible. Since each component of $P$ has nonempty boundary, this also implies that no component of $P$ is boundary parallel.

Lemma 6.2. Suppose $M=K(r)$ is Seifert fibered. Then there is an immersed torus or sphere $\hat{Q}$ in $M$ which is in essential position with respect to $P$.

Proof. By Lemma 6.1P is an embedded essential surface in $E\left(L_{r}\right)=E(L)$. Therefore the result follows from Lemma 4.5 if $\pi_{1}(K(r))$ is finite, and the surface $\hat{Q}$ is a sphere in this case.

Now assume $\pi_{1}(K(r))$ is infinite. By [Sc] a Seifert fibered manifold admits one of six 3-manifold geometries of Thurston. Since $K(r)$ is irreducible by Wu1, it does not admit $S^{2} \times R$ geometry, and since $\pi_{1}(K(r))$ is infinite it does not admit $S^{3}$ geometry. Hence the orbifold $X$ of $K(r)$ must be euclidean or hyperbolic and therefore has infinite orbifold fundamental group as defined in $\underline{\mathrm{Sc}}$. The torus $\hat{Q}$ in $K(r)$ that projects to a curve on $X$ with infinite order in the orbifold fundamental group is then an immersed torus in $K(r)$ which is $\pi_{1}$-injective in $K(r)$.

Let $Q=\hat{Q} \cap E\left(L_{r}\right)$. Choose $\hat{Q}$ to have minimal intersection with $L_{r}$ and then be homotoped so that $(|\partial Q \cap \partial P|,|Q \cap P|)$ is minimal in lexicographic order. In particular, $\partial Q$ intersects $\partial P$ minimally. We need to show that $\hat{Q}$ is in essential position with respect to $P$. Since $P$ is essential, we may get rid of loops in $Q \cap P$ which is trivial on $Q$. We need to show that all arc components $\alpha$ on $P \cap Q$ are nontrivial on both $P$ and $Q$. Note that $\alpha$ is embedded on $Q$ but may be immersed on $P$.

Assume $\alpha$ is inessential on both $P$ and $Q$. Then it is rel $\partial \alpha$ homotopic to an arc $\beta$ on $\partial Q$ and an $\operatorname{arc} \gamma$ on $\partial P$, so the incompressibility of $\partial N(K)$ implies that $\beta$ is 
homotopic to $\gamma$ and hence there is a homotopy to reduce $|\partial Q \cap \partial P|$, contradicting its minimality.

Now assume $\alpha$ is inessential on $P$ but essential on $Q$. Since $\alpha$ is trivial on $P$, we may deform $Q$ near $P$ to make $\alpha$ embedded on $P$. One can then push $\hat{Q}$ through the disk on $P$ cut off by $\alpha$ to reduce $\left|\hat{Q} \cap L_{r}\right|$ by 2 , contradicting its minimality.

If $\alpha$ is essential on $P$ but inessential on $Q$, then it cuts off a disk $D$ on $Q$ and hence is rel $\partial \alpha$ homotopic to the $\operatorname{arc} \partial D-\operatorname{Int} \alpha \subset \partial E\left(L_{r}\right)$. Since $P$ is essential by Lemma 6.1, this contradicts Lemma 4.1.

Let $\hat{Q}$ be as in Lemma 6.2 and put $Q=\hat{Q} \cap E\left(L_{r}\right)$. Since $P$ is essential and embedded, $P \cap Q$ is an embedded compact 1-manifold in $Q$. However it may not be embedded in $P$ because $Q$ is immersed in $E(L)$. Since $\hat{Q}$ is in essential position with respect to $P$, no component of $Q \cap P$ is a trivial circle or trivial arc on $Q$.

Define a generalized graph to be a graph with possibly some valence 2 vertices removed from the vertex list. Thus it is a graph except that some loop components may not have vertices on it.

Definition 6.3. Consider the disks of $\hat{Q}-\operatorname{Int} Q=\hat{Q} \cap N\left(L_{r}\right)$ as fat vertices, the arc components of $P \cap Q$ as edges, and the circles of $P \cap Q$ as loops. This produces a generalized graph $G$ on $\hat{Q}$, called the intersection graph of $\hat{Q}$ and $P$.

There are two types of vertices on $G$. A component of $\hat{Q} \cap N(C)$ is called a small vertex, and a component of $\hat{Q} \cap N(K)$ is called a large vertex. Since a meridian of $C$ intersects $P$ at three points, one on each $P_{i}$, we see that each small vertex has valence 3 . Denote by $\Delta=\Delta(\mu, r)$ the minimal intersection number between a meridian $\mu$ of $K$ and the surgery slope $r$. Then the surgery slope $r$ intersects $\partial P$ minimally at $6 \Delta$ points, so each large vertex has valence $6 \Delta$, with $2 \Delta$ endpoints on each $P_{i}$. In particular, if $r$ is an integer slope, we have $\Delta=1$, in which case the valence of each large vertex is 6 .

Write the boundary of the tangle space $E\left(t_{i}\right)$ as $\partial E\left(t_{i}\right)=P_{i} \cup P_{i+1} \cup A_{i} \cup A_{i}^{\prime} \cup A_{i}^{\prime \prime}$, where $P_{i}$ are the twice-punctured disk $D_{i} \cap E(L)$ defined above, $A_{i}=\partial N(C) \cap E\left(t_{i}\right)$, and $A_{i}^{\prime}, A_{i}^{\prime \prime}$ are the two annuli $\partial N(K) \cap E\left(t_{i}\right)$. Since $\hat{Q}$ is in essential position with $P$, we have the following lemma.

Lemma 6.4. Let $\sigma$ be a face of $G$ lying in $E\left(t_{i}\right)$. Then $\partial \sigma$ intersects each of the above five subsurfaces of $\partial E\left(t_{i}\right)$ in essential arcs and hence is a set of tight curves. In particular, each disk face of $G$ is an essential disk in some $E\left(t_{i}\right)$.

An arc of a face $\sigma$ on the boundary of a fat vertex $v$ is called a corner of $\sigma$ at $v$. Note that when shrinking each fat vertex to a single point, a corner becomes a vertex on the boundary of the face $\sigma$. A corner at $v$ is large or small according to whether $v$ is a large vertex or a small vertex. Thus a large corner lies on $A_{i}^{\prime}$ or $A_{i}^{\prime \prime}$ for some $i$ while a small corner is on the other annulus $A_{i}$ and hence intersects $m_{i}$ at a single point. Therefore a disk face $\sigma$ of $G$ is of type $(r, s)$ if and only if it has $r$ large corners and $s$ small corners. The results in Section 3 now apply to the disk faces of $G$. In particular, an $(r, s)$ face in $E\left(t_{i}\right)$ has $s \geq 2 q_{i}$ if $r=0, s \geq q_{i}$ if $r$ is odd, and $s \geq 2\left|\bar{p}_{i}\right|$ if $r$ is even.

\section{EULER NUMBER OF AN ANGLED SURFACE}

An angled surface is a compact surface $\sigma$ with a set of points $V=\left(v_{1}, \ldots, v_{n}\right)$ on $\partial \sigma$ called vertices or corners, and an angle $\alpha_{i}$ assigned to each corner $v_{i}$, with 
$0 \leq \alpha_{i}<\pi$. When $\sigma$ is a disk, it is a polygon or $n$-gon. We will always use $\bar{\alpha}$ to denote the external angle $\bar{\alpha}=\pi-\alpha$.

Definition 7.1. The angled Euler number of an angled surface $\sigma$ with corner angles $\alpha_{1}, \ldots, \alpha_{n}$ is defined as

$$
e(\sigma)=\chi(\sigma)-\frac{1}{2 \pi} \sum \bar{\alpha}_{i}
$$

Recall that a generalized graph is a graph on which some of the loops may not have vertices.

Lemma 7.2. Let $G$ be a generalized graph on a closed surface $F$, cutting it into angled surfaces $\sigma_{1}, \ldots, \sigma_{m}$, such that the sum of angles around each vertex is at least $2 \pi$. Then $\chi(F) \leq \sum e\left(\sigma_{i}\right)$, and equality holds if and only if the sum of angles around each vertex is $2 \pi$.

Proof. Note that adding vertices to loops with an angle $\pi$ at each corner of the new vertices will not change the angled Euler number of the faces. Therefore by adding such vertices if necessary we may assume that $G$ is a genuine graph. We assume that the sum of the angles around each vertex of $G$ is exactly $2 \pi$. The proof for the other case is similar.

Let $n_{i}$ be the number of vertices on $\partial \sigma_{i}$, let $v_{i j}$ be the vertices on $\partial \sigma_{i}$, and let $\alpha_{i j}$ be the angles at $v_{i j}$. Note that $n_{i}$ is also the number of edges on $\partial \sigma_{i}$. Denote by $E$ and $V$ the number of edges and vertices of $G$, respectively. Then

$$
\begin{aligned}
\sum_{i} e\left(\sigma_{i}\right) & =\sum_{i}\left[\chi\left(\sigma_{i}\right)-\frac{1}{2 \pi} \sum_{j}\left(\pi-\alpha_{i j}\right)\right] \\
& =\sum_{i}\left[\chi\left(\sigma_{i}\right)-\frac{1}{2} n_{i}+\sum_{j} \frac{\alpha_{i j}}{2 \pi}\right] \\
& =\sum \chi\left(\sigma_{i}\right)-\sum \frac{n_{i}}{2}+\frac{\sum \alpha_{i j}}{2 \pi} \\
& =\sum \chi\left(\sigma_{i}\right)-E+V \\
& =\chi(F) .
\end{aligned}
$$

A face $\sigma$ is said to be spherical, euclidean, or hyperbolic according to whether $e(\sigma)$ is positive, zero, or negative, respectively. When the angles are nonzero, one can show that $\sigma$ has a corresponding geometric structure with geodesic boundary edges and an angle of $\alpha_{i}$ at corner $v_{i}$. Thus for example if all $\sigma_{i}$ are euclidean or hyperbolic with at least one $\sigma_{i}$ hyperbolic, and if the sum of angles at each vertex is at least $2 \pi$, then the surface $F$ is a hyperbolic surface.

\section{Proof of the MAIN THEOREMS}

Suppose $K(r)$ is an atoroidal Seifert fiber space. By Lemma 6.2 there is an immersed sphere or a torus $\hat{F}$ in $K(r)$ which is in essential position with respect to $P=P_{1} \cup P_{2} \cup P_{3}$. Let $G$ be the generalized graph on $\hat{F}$ as defined in Section 6. Let $\alpha_{i}$ and $\beta_{i}$ be the angles of large corners and small corners of $F \cap E\left(t_{i}\right)$, respectively. We would like to show that if $\alpha_{i}, \beta_{i}$ can be chosen to satisfy certain conditions, then $K(r)$ must be toroidal.

As before, denote by $\bar{\alpha}_{i}=\pi-\alpha_{i}$ and $\bar{\beta}_{i}=\pi-\beta_{i}$. Recall that $\bar{p}_{i}$ is the $\bmod q_{i}$ inverse of $-p_{i}$, as in Definition 2.2. Let $\mu$ be the meridional slope of $K$. 
Theorem 8.1. Suppose $K=K\left(p_{1} / q_{1}, p_{2} / q_{2}, p_{3} / q_{3}\right)$ is a hyperbolic Montesinos knot with $q_{i} \geq 2$. Then $K(r)$ is not an atoroidal Seifert fibered manifold if there are angles $\pi \geq \bar{\alpha}_{i}>0$ and $\pi>\bar{\beta}_{i}>0$ satisfying the following conditions:

(1) $\bar{\alpha}_{1}+\bar{\alpha}_{2}+\bar{\alpha}_{3} \leq 2 \pi$;

(2) $\bar{\beta}_{1}+\bar{\beta}_{2}+\bar{\beta}_{3} \leq \pi$

(3) $\bar{\alpha}_{i}+q_{i} \bar{\beta}_{i} \geq 2 \pi$;

(4) $\bar{\alpha}_{i}+\left|\bar{p}_{i}\right| \bar{\beta}_{i} \geq \pi$

(5) if $q_{i}=2$, then $\bar{\alpha}_{i}+\bar{\beta}_{i}>\pi$.

Proof. Assume to the contrary that $K(r)$ is an atoroidal Seifert fibred manifold. Let $\hat{F}$ and $F$ be as above, with $\alpha_{i}$ and $\beta_{i}$ the large corners and small corners of $F \cap E\left(t_{i}\right)$, respectively. Conditions (1) and (2) can be rewritten as $\sum 2 \alpha_{i} \geq 2 \pi$ and $\sum \beta_{i} \geq 2 \pi$, which mean that the sum of the angles around each vertex of $G$ is at least $2 \pi$. Conditions (3) and (4) say that the sum of external angles of a face $\sigma$ of type $(1, q)$ or $\left(2,2\left|\bar{p}_{i}\right|\right)$ is at least $2 \pi$, so by definition we have $e(\sigma) \leq 0$. We want to show that $e(\sigma) \leq 0$ for all other faces as well.

By Lemmas 3.3 and 3.4 a disk face of type $(r, s)$ in $E\left(t_{i}\right)$ is of one of the following types:

(a) $r=0$ and $s \geq 2 q_{i}$;

(b) $r$ is odd and $s \geq q_{i}$;

(c) $r \geq 2$ is even and $s \geq 2\left|\bar{p}_{i}\right|$.

Let $\sigma_{j}$ be a face of type $j=a, b$, or $c$ above in $E\left(t_{i}\right)$. Then

$$
\begin{aligned}
& e\left(\sigma_{a}\right)=1-\frac{1}{2 \pi} s \bar{\beta}_{i} \leq \frac{2}{2 \pi}\left(\pi-q_{i} \bar{\beta}_{i}\right) \leq \frac{\bar{\alpha}_{i}-\pi}{\pi} \leq 0, \\
& e\left(\sigma_{b}\right)=1-\frac{1}{2 \pi}\left(r \bar{\alpha}_{i}+s \bar{\beta}_{i}\right) \leq 1-\frac{1}{2 \pi}\left(\bar{\alpha}_{i}+q_{i} \bar{\beta}_{i}\right) \leq 0, \\
& e\left(\sigma_{c}\right)=1-\frac{1}{2 \pi}\left(r \bar{\alpha}_{i}+s \bar{\beta}_{i}\right) \leq 1-\frac{1}{2 \pi}\left(2 \bar{\alpha}_{i}+2\left|\bar{p}_{i}\right| \bar{\beta}_{i}\right) \leq 0 .
\end{aligned}
$$

By definition all nondisk faces $\sigma$ of $G$ have $e(\sigma) \leq 0$. Thus all faces $\sigma$ of $G$ have $e(\sigma) \leq 0$. Since $\chi(\hat{F})=\sum e(\sigma) \leq 0$ by Lemma 7.2, the surface $\hat{F}$ cannot be a sphere, so it must be a torus, and $e(\sigma)=0$ for all faces $\sigma$. If there is a nondisk face, then the outermost one has some corner on it and hence has $e(\sigma)<0$, which is a contradiction. Therefore all $\sigma$ are disk faces with $e(\sigma)=0$. This implies that all the above inequalities must be equalities. Since $\bar{\alpha}_{i}, \bar{\beta}_{i}>0$, checking the above inequalities gives the following.

(a) If $\sigma_{a}$ exists in $E\left(t_{i}\right)$, then $\bar{\alpha}_{i}=\pi$ and $s=2 q_{i}$, so $\sigma_{a}$ is a $\left(0,2 q_{i}\right)$ face.

(b) If $\sigma_{b}$ exists in $E\left(t_{i}\right)$, then $r=1$ and $s=q_{i}$, so $\sigma_{b}$ is a $\left(1, q_{i}\right)$ face.

(c) If $\sigma_{c}$ exists in $E\left(t_{i}\right)$, then $r=2$ and $s=2\left|\bar{p}_{i}\right|$, so $\sigma_{c}$ is a $\left(2,2\left|\bar{p}_{i}\right|\right)$ face.

These imply that $F$ satisfies condition (1) in Definition 5.1. Condition (1) above implies that $\bar{\alpha}_{i}<\pi$ for some $i$, hence (a) above implies that there is no face of type $\left(0,2 q_{i}\right)$ in $F \cap E\left(t_{i}\right)$ for some $i$, so condition (2) in Definition 5.1 holds. When $q_{i}=2$ we have $\bar{p}_{i}=1$, and by condition (5) above we have $\bar{\alpha}_{i}+\bar{\beta}_{i}>\pi$, so by the calculation above we would have $e\left(\sigma_{c}\right)<0$, which is a contradiction. Therefore there is no disk of type $(2,2)=\left(2,2\left|\bar{p}_{i}\right|\right)$, which gives (3) in Definition 5.1. Similarly if $F \cap E\left(t_{i}\right)$ has a disk of type $\left(0,2 q_{i}\right)$, then $e\left(\sigma_{a}\right)=0$ gives $\bar{\alpha}_{i}=\pi$, and again we have $\bar{\alpha}_{i}+\left|\bar{p}_{i}\right| \bar{\beta}_{i}>\pi$, hence there is no disk of type $\left(2,2\left|p_{i}\right|\right)$, which verifies condition (4) of Definition 5.1. Therefore $F$ is an elementary surface. It now follows from Proposition 5.6 that $K(r)$ is toroidal, a contradiction. 
Proof of Theorem 1.1. Let $K=K\left(p_{1} / q_{1}, p_{2} / q_{2}, p_{3} / q_{3}\right)$, with $2 \leq q_{1} \leq q_{2} \leq q_{3}$. Since $K$ is a knot, at most one $q_{i}$ is even. Hence the condition $\sum \frac{1}{q_{i}-1} \leq 1$ implies that either $q_{i} \geq 4$ for all $i$, or $q_{1}=3$ and $q_{2}, q_{3} \geq 5$, or $q_{1}=3, q_{2}=4$ and $q_{3} \geq 7$.

If $q_{i} \geq 4$, let $\bar{\alpha}_{1}=\bar{\alpha}_{2}=\bar{\alpha}_{3}=\frac{2 \pi}{3}$ and $\bar{\beta}_{1}=\bar{\beta}_{2}=\bar{\beta}_{3}=\frac{\pi}{3}$.

If $q_{1}=3$ and $q_{2}, q_{3} \geq 5$, let $\left(\bar{\alpha}_{1}, \bar{\alpha}_{2}, \bar{\alpha}_{3}\right)=\left(\frac{\pi}{2}, \frac{3 \pi}{4}, \frac{3 \pi}{4}\right)$ and $\left(\bar{\beta}_{1}, \bar{\beta}_{2}, \bar{\beta}_{3}\right)=\left(\frac{\pi}{2}, \frac{\pi}{4}, \frac{\pi}{4}\right)$.

If $q_{1}=3, q_{2}=4$ and $q_{3} \geq 7$, let $\left(\bar{\alpha}_{1}, \bar{\alpha}_{2}, \bar{\alpha}_{3}\right)=\left(\frac{\pi}{2}, \frac{2 \pi}{3}, \frac{5 \pi}{6}\right)$ and $\left(\beta_{1}, \beta_{2}, \beta_{3}\right)=$ $\left(\frac{\pi}{2}, \frac{\pi}{3}, \frac{\pi}{6}\right)$.

One can easily check that the conditions (1)-(3) of Theorem 8.1 are satisfied in each of the above cases. Note that in all cases we have $\bar{\alpha}_{i}+\bar{\beta}_{i}=\pi$, so condition (4) holds because $\left|\bar{p}_{i}\right| \geq 1$. Condition (5) holds trivially since $q_{i}>2$. Theorem 1.1 now follows from Theorem 8.1.

By Theorem 1.1 if a Montesinos knot $K$ of length 3 admits atoroidal Seifert fibered surgery, then $K=K\left(\frac{p_{1}}{q_{1}}, \frac{p_{2}}{q_{2}}, \frac{p_{3}}{q_{3}}\right)$, such that either $q_{1}=2$, or $\left(q_{1}, q_{2}\right)=(3,3)$ or $\left(q_{1}, q_{2}, q_{3}\right)=(3,4,5)$. The following theorem gives further restrictions on $p_{i}$. In this theorem it is not assumed that $q_{2} \leq q_{3}$, so $q_{2}$ in (4) below may be larger than $q_{3}$. Two knots $K, K^{\prime}$ are equivalent if $K$ is isotopic to $K^{\prime}$ or its mirror image.

Theorem 8.2. Suppose a Montesinos knot $K$ of length 3 admits an atoroidal Seifert fibered surgery. Then $K$ is equivalent to one of the following knots:

(1) $K\left(1 / 3, \pm 1 / 4, p_{3} / 5\right)$ and $p_{3} \equiv \pm 1 \bmod 5$;

(2) $K\left(1 / 3, \pm 1 / 3, p_{3} / q_{3}\right)$ and $\left|\bar{p}_{3}\right| \leq 2$;

(3) $K\left(1 / 2,2 / 5, p_{3} / q_{3}\right), q_{3}=5$ or 7 , and $\left|\bar{p}_{3}\right|>1$;

(4) $K\left(1 / 2,1 / q_{2}, p_{3} / q_{3}\right), q_{2} \geq 5$ and $\left|\bar{p}_{3}\right| \leq 2$;

(5) $K\left(1 / 2,1 / 3, p_{3} / q_{3}\right)$ and $\left|\bar{p}_{3}\right| \leq 6$.

Proof. By Theorem 1.1, we have $\left(q_{1}, q_{2}, q_{3}\right)=(3,4,5),\left(3,3, q_{3}\right)$ or $\left(2, q_{2}, q_{3}\right)$. It is easy to see that if $K$ is not equivalent to one of those listed in the theorem, then it is listed in one of the five cases below. In each case we will list the angles $\bar{\alpha}_{i}, \bar{\beta}_{i}$. These satisfy $\sum \bar{\alpha}_{i}=2 \pi, \sum \bar{\beta}_{i}=\pi$, and $\alpha_{1}=\pi$ when $q_{1}=2$, so conditions (1), (2) and (5) in Theorem 8.1 hold. We leave it to the reader to check that they satisfy conditions $(3) \bar{\alpha}_{i}+q_{i} \bar{\beta}_{i} \geq 2 \pi$ and (4) $\bar{\alpha}_{i}+\left|\bar{p}_{i}\right| \bar{\beta}_{i} \geq \pi$ in Theorem 8.1.

Case 1. $\left(q_{1}, q_{2}, q_{3}\right)=(3,4,5)$, and $\left|\bar{p}_{3}\right|=2$.

Let $\left(\bar{\alpha}_{1}, \bar{\alpha}_{2}, \bar{\alpha}_{3}\right)=\left(\pi, \frac{2 \pi}{3}, \frac{\pi}{3}\right)$ and $\left(\bar{\beta}_{1}, \bar{\beta}_{2}, \bar{\beta}_{3}\right)=\left(\frac{\pi}{3}, \frac{\pi}{3}, \frac{\pi}{3}\right)$.

Case 2. $q_{1}=q_{2}=3$, and $\left|\bar{p}_{3}\right| \geq 3$.

We must have $q_{3} \geq 7$ because $2\left|\bar{p}_{3}\right| \leq q_{3}$ and $\bar{p}_{3}$ is coprime with $q_{3}$. Let $\left(\bar{\alpha}_{1}, \bar{\alpha}_{2}, \bar{\alpha}_{3}\right)=\left(\frac{7 \pi}{8}, \frac{7 \pi}{8}, \frac{\pi}{4}\right)$ and $\left(\bar{\beta}_{1}, \bar{\beta}_{2}, \bar{\beta}_{3}\right)=\left(\frac{3 \pi}{8}, \frac{3 \pi}{8}, \frac{\pi}{4}\right)$.

Case $3 . q_{1}=2$, and $\left|\bar{p}_{i}\right|>1$ for $i=2,3$.

Note that $q_{2}, q_{3}$ must be odd since $K$ is a knot. By definition of $\left|\bar{p}_{i}\right|$ we have $q_{2}, q_{3} \geq 5$. If $q_{2}, q_{3} \geq 7$, let $\left(\bar{\alpha}_{1}, \bar{\alpha}_{2}, \bar{\alpha}_{3}\right)=\left(\pi, \frac{\pi}{2}, \frac{\pi}{2}\right)$ and $\left(\bar{\beta}_{1}, \bar{\beta}_{2}, \bar{\beta}_{3}\right)=\left(\frac{\pi}{2}, \frac{\pi}{4}, \frac{\pi}{4}\right)$. If $q_{2}=5$ and $q_{3} \geq 9$, let $\left(\bar{\alpha}_{1}, \bar{\alpha}_{2}, \bar{\alpha}_{3}\right)=\left(\pi, \frac{\pi}{3}, \frac{2 \pi}{3}\right)$ and $\left(\bar{\beta}_{1}, \bar{\beta}_{2}, \bar{\beta}_{3}\right)=\left(\frac{\pi}{2}, \frac{\pi}{3}, \frac{\pi}{6}\right)$. Both cases can be excluded by Theorem 8.1. Therefore up to equivalence we have $q_{2}=5$ and $q_{3}=5$ or 7 , as in (3).

Case 4. $q_{1}=2,\left|\bar{p}_{2}\right|=1, q_{2} \geq 5,\left|\bar{p}_{3}\right| \geq 3$.

By convention we have $2\left|p_{i}\right| \leq q_{i}$ for $i=1,2$, hence $p_{2}= \pm 1$. The condition $\left|\bar{p}_{3}\right| \geq 3$ implies that $q_{3} \geq 7$. Let $\left(\bar{\alpha}_{1}, \bar{\alpha}_{2}, \bar{\alpha}_{3}\right)=\left(\pi, \frac{3 \pi}{4}, \frac{\pi}{4}\right)$ and $\left(\bar{\beta}_{1}, \bar{\beta}_{2}, \bar{\beta}_{3}\right)=$ $\left(\frac{\pi}{2}, \frac{\pi}{4}, \frac{\pi}{4}\right)$. 
Case 5. $q_{1}=2, q_{2}=3,\left|\bar{p}_{3}\right| \geq 7$.

We have $q_{3} \geq 15$. Let $\left(\bar{\alpha}_{1}, \bar{\alpha}_{2}, \bar{\alpha}_{3}\right)=\left(\pi, \frac{7 \pi}{8}, \frac{\pi}{8}\right)$ and $\left(\bar{\beta}_{1}, \bar{\beta}_{2}, \bar{\beta}_{3}\right)=\left(\frac{\pi}{2}, \frac{3 \pi}{8}, \frac{\pi}{8}\right)$.

In all cases above, we see that $\bar{\alpha}_{i}, \bar{\beta}_{i}$ satisfy all conditions of Theorem 8.1, therefore by that theorem $K(r)$ is not an atoroidal Seifert fibred manifold, a contradiction.

Remark 8.3. The first two tangles of the knots in the above theorem are very simple. The small value of $\left|\bar{p}_{3}\right|$ implies that the third tangle of those knots are also relatively simple. For example if $\bar{p}_{3}=2$ and if we write $p_{3}=n q_{3}+m$ with $2|m|<q_{3}$, then $p_{3} \bar{p}_{3} \equiv m \bar{p}_{3} \equiv-1 \bmod q_{3}$ implies that $q_{3}= \pm\left(m \bar{p}_{3}+1\right)= \pm 2 m \pm 1$, so we have a partial fraction decomposition $p_{3} / q_{3}=n \pm 1 /(2 \pm 1 / m)$. The results above will be used in Wu4 to reduce the classification problem of Seifert fibered surgeries on Montesinos knots to that on a few specific families of knots.

\section{REFERENCES}

[BW] M. Brittenham and Y-Q. Wu, The classification of exceptional Dehn surgeries on 2bridge knots, Comm. Anal. Geom. 9 (2001), 97-113. MR1807953 (2001m:57008)

[De] C. Delman, Constructing essential laminations which survive all Dehn surgeries, preprint.

[Ga] D. Gabai, Foliations and the topology of 3-manifolds III, J. Diff. Geom. 26 (1987), 479-536. MR910018(89a:57014b)

[FIKMS] D. Futer, M. Ishikawa, Y. Kabaya, T. Mattman and K. Shimokawa, Finite surgeries on three-tangle pretzel knots, Algebr. Geom. Topol. 9 (2009), 743-771. MR2496889 (2010a:57008)

[GW] C. Gordon and Y-Q. Wu, Annular Dehn fillings, Comment. Math. Helv. 75 (2000), 430-456. MR1793797 (2001j:57024)

[HO] A. Hatcher and U. Oertel, Boundary slopes for Montesinos knots, Topology 28 (1989), 453-480. MR1030987 (91e:57016)

[HT] A. Hatcher and W. Thurston, Incompressible surfaces in 2-bridge knot complements, Invent. Math. 79 (1985), 225-246. MR778125 (86g:57003)

[IJ1] K. Ichihara, I. Jong, Cyclic and finite surgeries on Montesinos knots, Alg. Geom. Topol. 9 (2009), 731-742. MR2496888(2011b:57021)

[IJ2] _ Toroidal Seifert fibered surgeries on Montesinos knots, Comm. Anal. Geom. 18 (2010), 579-600. MR2747439 (2012f:57040)

[Ma] T. Mattman, Cyclic and finite surgeries on pretzel knots, J. Knot Theory Ram. 11 (2002), 891-902. MR 1936241 (2003j:57013)

[Ni] Y. Ni, Knot Floer homology detects fibred knots, Invent. Math. 170 (2007), 577-608. MR2357503 (2008j:57053)

[Pr] G. Perelman, The entropy formula for the Ricci flow and its geometric applications, ArXiv:math.DG/0211159.

[Sc] P. Scott, The geometries of 3-manifolds, Bull. London Math. Soc. 15 (1983), 401-487. MR705527 (84m:57009)

[Wa] L. Watson, Surgery obstructions from Khovanov homology, preprint.

[Wu1] Y-Q. Wu, Dehn surgery on arborescent knots, J. Diff. Geom. 42 (1996), 171-197. MR 1424423 (97j:57013)

[Wu2] _ Exceptional Dehn surgery on large arborescent knots, Pac. J. Math. 252 (2011), 219-243. MR2862149

[Wu3] , The classification of toroidal Dehn surgeries on Montesinos knots, Comm. Anal. Geom. 19 (2011), 305-345. MR2835882

[Wu4] _ Persistently laminar branched surfaces, Comm. Anal. Geom. 20 (2012), 397434.

Department of Mathematics, University of Iowa, Iowa City, Iowa 52242

E-mail address: wu@math.uiowa.edu 